\title{
Comunicación
}

\section{Enterotomía revestida con ligamento ileocecal en un equino: reporte de caso}

\author{
Enterotomy covered with ileocecal ligament in an equine: case report
}

\author{
Gabriel Gómez D. ${ }^{1}$, Ceesar Aguilar G.,3, Cristina García B. ${ }^{2}$, Alfredo Delgado C. ${ }^{2}$
}

\section{Resumen}

Se reporta el caso de un equino hembra de raza Pura Sangre de Carrera de tres años con síndrome abdominal agudo relacionado a una hernia umbilical irreductible, inflamada y tumefacta, que fue derivada a cirugía. Durante el procedimiento quirúrgico se detectó una adherencia necrótica en la parte antimesentérica de un segmento del íleon. Con el fin de extraer la porción afectada se practicó una enterotomía, la cual fue reforzada mediante la transposición del ligamento ileocecal para evitar una estenosis o el posible colapso de la línea anastomótica. Los resultados de la cirugía fueron satisfactorios y el paciente evolucionó favorablemente.

Palabras clave: hernia umbilical; adherencia; enterotomía; revestimiento; hernia de Richter

\section{Abstract}

The case of a three-year-old Thoroughbred female horse with acute abdominal syndrome related to an irreducible, swollen and swollen umbilical hernia, which was referred to surgery is reported. During the surgical procedure a necrotic adhesion was detected in the antimesenteric part of a segment of the ileum. In order to remove

\footnotetext{
${ }^{1}$ Servicio de Veterinaria y Remonta del Ejército, Uruguay.

${ }^{2}$ Clínica de Animales Mayores, Facultad de Medicina Veterinaria - Universidad Nacional Mayor de San Marcos, Lima, Perú

${ }^{3}$ E-mail: ceniague@gmail.com
}

Recibido: 16 de febrero de 2019

Aceptado para publicación: 23 de octubre de 2019 
the affected portion, an enterotomy was performed, which was reinforced by transposition of the ileocecal ligament to avoid stenosis or the possible collapse of the anastomotic line. The results of the surgery were satisfactory, and the patient evolved favourably.

Key words: umbilical hernia; adherence; enterotomy; coating; Richter hernia

\section{INTRODUCCIÓN}

El término hernia hace referencia a la protrusión de la pared de una cavidad pudiendo albergar a uno o varios órganos (Tóth y Schumacher, 2019). Las hernias umbilicales son el tipo más común de hernia y el segundo defecto congénito más común en equinos, con una frecuencia de presentación entre $0.5 \mathrm{y}$ 2\% (Lavoie y Hinchcliff, 2008; Neil, 2011). Entre las complicaciones que pueden presentarse se pueden mencionar la estrangulación, incarceramiento, fístulas enterocutáneas, abscesos, adherencias y hernias parietales o de Richter (Neil, 2011; Wilson, 2012; Smith, 2015). La hernia parietal o de Richter se define como una hernia abdominal en la que solo una parte de la pared antimesentérica del intestino está incarcerada en el saco herniario (Steinke y Zellweger, 2000; Martis et al., 2011), siendo la porción inferior del íleon el segmento intestinal más comprometido (Smith, 2006; Martis et al., 2011).

El atrapamiento de una parte de la circunferencia intestinal puede dar lugar a necrosis del segmento herniado con la consiguiente formación de abscesos y fístulas (Bristol, 1994). El diagnóstico se realiza mediante examen clínico, y se sospecha de una hernia de Richter cuando una hernia umbilical se vuelve irreductible, grande, firme, edematosa y dolorosa a la palpación (Kummer y Stick, 2012). Además, equinos con este tipo de hernia pueden desarrollar episodios de cólico (Freeman, 2019). El tratamiento es quirúrgico, debiendo realizar la resección del segmento intestinal incancerado de estar comprometida su viabilidad (Ramírez, 2013).

\section{Caso Clínico}

\section{Historia Clínica}

Una potranca de tres años de raza Pura Sangre de Carrera (PSC) en doma presentaba signología de Síndrome Abdominal Agudo (SAA) con 48 horas de evolución. La paciente presentaba dolores controlables con dipirona el primer día de presentación del cuadro, pero pasadas 24 horas fue necesario brindar analgesia con dosis de $1 \mathrm{mg} / \mathrm{kg}$ de flunixin meglumina. Al examen clínico, la potranca presentaba una frecuencia cardiaca (FC) de 48 lpm, temperatura rectal normal, leve deshidratación, disminución de apetito y aparente congestión de las mucosas orales. Además, presentaba una hernia umbilical no reparada (el propietario la adquirió con la hernia de destete a los seis meses de edad y siempre estuvo en pastura y con concentrados una vez al día hasta la doma, sin signos locales). Aparentemente, la hernia se hizo irreductible, dolorosa a la palpación y con evidente tumefacción inflamatoria durante el curso del SAA.

Al día siguiente de la consulta, el paciente fue derivado al Hospital Quirúrgico Veterinario del Servicio de Veterinaria y Remonta del Ejército, Canelones, Uruguay. Al examen clínico, la potranca no evidenciaba signos de dolor abdominal, pero presentaba una evidente deformación ventral a la altura del ombligo, con importante tumefacción y dolor a la palpación, dificultando la realización de un estudio ultrasonográfico de la zona. El animal se encontraba con una FC de 54, buena hidratación y sonidos abdominales re- 

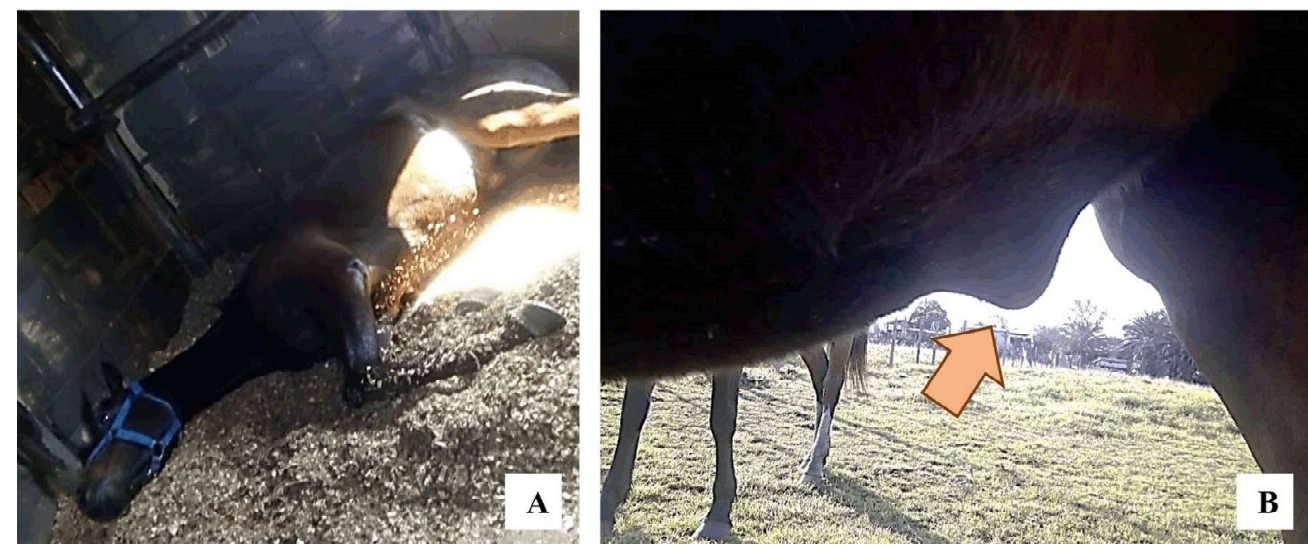

Figura 1. Potranca Pura Sangre de Carrera de tres años con signos de Síndrome Abdominal Agudo (SAA) con 48 horas de evolución. A) Episodio de cólico del paciente; B) hernia umbilical (vista lateral)

ducidos en ambos flancos; por lo cual, se decidió a proceder con la intervención quirúrgica inmediata.

\section{Técnica Anestésica}

El procedimiento anestésico fue realizado mediante un derribo farmacológico (1.1 $\mathrm{mg} / \mathrm{kg}$ de xilacina $10 \%$ (Xilamic, Laboratorios Microsules, Uruguay), $0.15 \mathrm{mg} / \mathrm{kg}$ de diazepam 5\% (Dazam, Richmond Veterinaria, Argentina) y $2.2 \mathrm{mg} / \mathrm{kg}$ de ketamina al 5\% (Ketonal 50, Richmond Veterinaria, Argentina) y mantenimiento de forma intravenosa (IV) (triple goteo: $1 \mathrm{~g}$ de xilacina $+2 \mathrm{~g}$ ketamina $+50 \mathrm{~g}$ éter glicerilguayacólico (Éter Gliceril Guayacólico, Pro-Ser, Argentina) en $1 \mathrm{~L}$ de suero glucosado al $5 \%$ ) con intubación endotraqueal y administración de 5 1/min de oxígeno al $100 \%$. Fluidoterapia IV con ringer lactato durante todo el procedimiento.

\section{Procedimiento Quirúrgico}

El paciente fue ubicado en decúbito dorsal, realizando el abordaje por línea media. Se procedió a drenar el absceso umbilical subcutáneo al inicio de la apertura de la línea media, donde se encontró gran depósito de

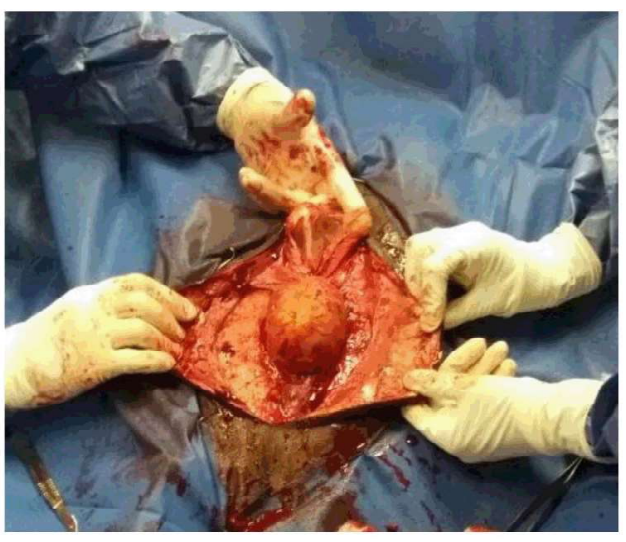

Figura 2. Exposición de la bolsa herniaria con contenido purulento en una potranca Pura Sangre de Carrera

fibrina alrededor del saco herniario. Así mismo, estaba adherido a una parte de la cara antimesentérica del íleon (Figura 2).

Debido a que el tiempo quirúrgico estaba condicionado al corto periodo anestésico, no se podía realizar la anastomosis yeyunocecal. En consecuencia, al encontrar que la necrosis del segmento adherido se limitaba a $4 \mathrm{~cm}$ de diámetro en la cara antimesentérica (Figura 3), se decidió realizar una escisión 

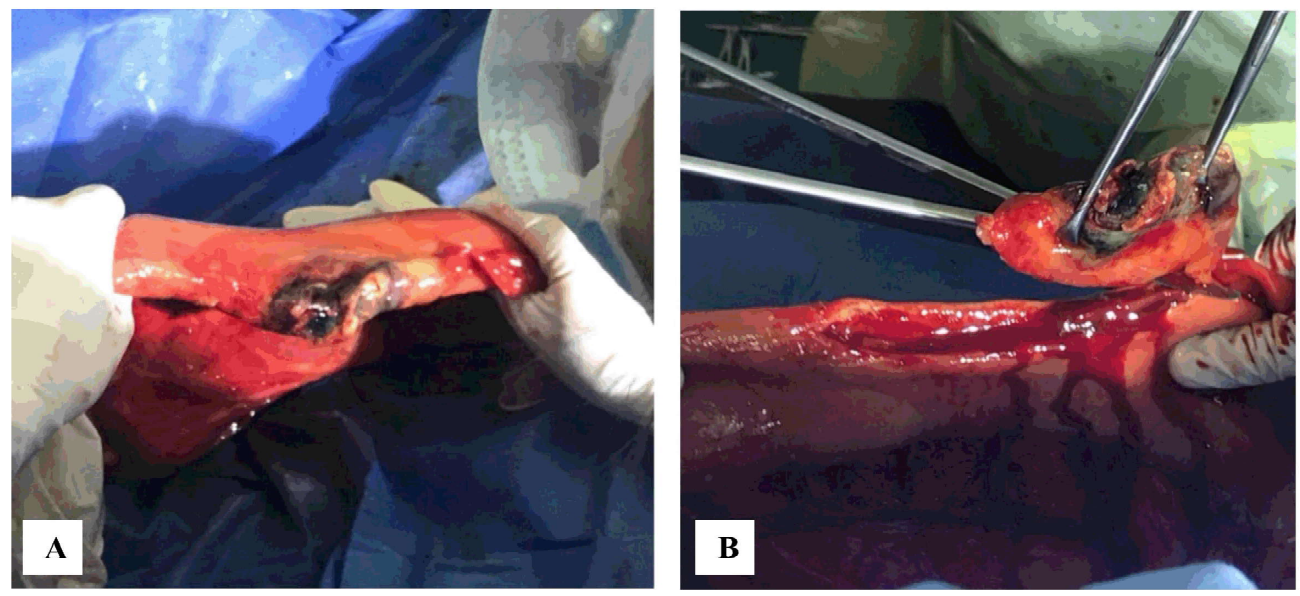

Figura 3. Zona ileal necrótica adherida al saco herniario (A); exéresis del área mediante enterotomía en escisión elíptica longitudinal (B) en una potranca Pura Sangre de Carrera
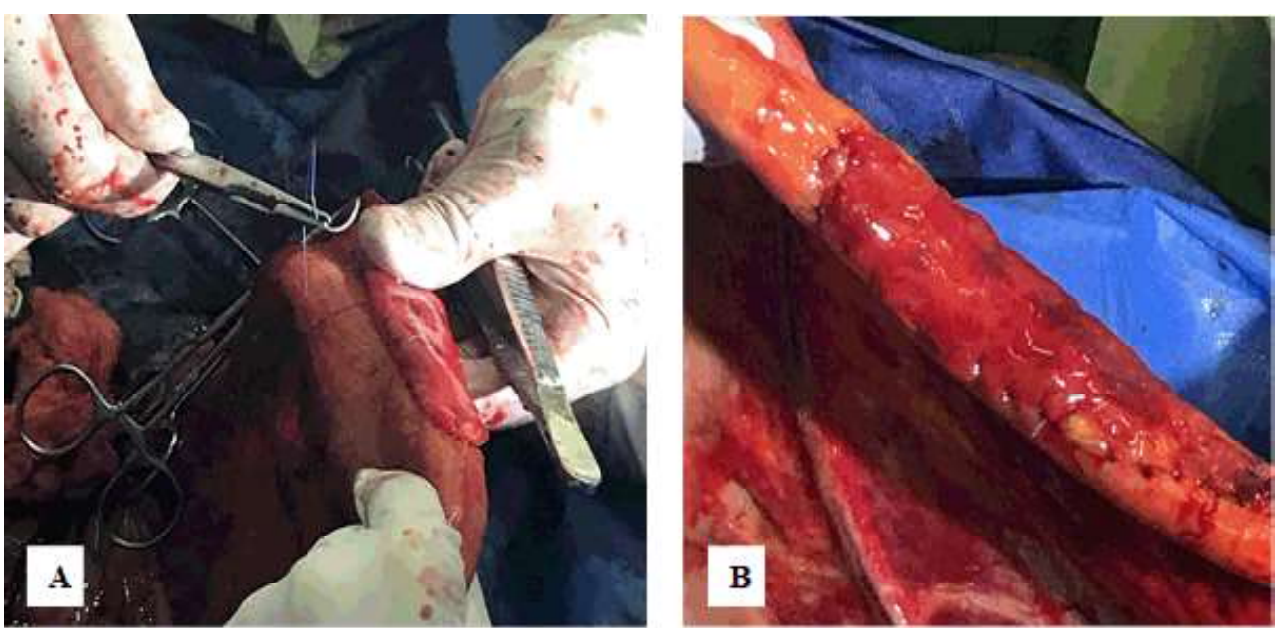

Figura 4.Revestimiento de la línea de sutura con trasposición del ligamento ileocecal (A); enterotomía con revestimiento completada (B), en una potranca Pura Sangre de Carrera

elíptica longitudinal del área mediante enterotomía. La considerable resección de tejido no permitió la aplicación de un patrón de sutura invaginante en la línea de enterotomía, debido al riesgo de generar estenosis de la luz intestinal y a la imposibilidad de ejercer una buena tensión de la sutura a causa del evidente edema subseroso. Se decidió por una sutura continua con puntos simples perforantes (vycril N. ${ }^{\circ} 0$ ), la cual se revis- tió transponiendo un segmento del ligamento ileocecal a modo de parche (vycril N. ${ }^{\circ} 2-0$ ) (Figura 4). El cierre de la pared abdominal se realizó mediante una sutura simple continua con hilo nylon monofilamento.

\section{Tratamiento Posoperatorio}

Al finalizar el procedimiento quirúrgico se procedió a la recuperación anestésica asis- 

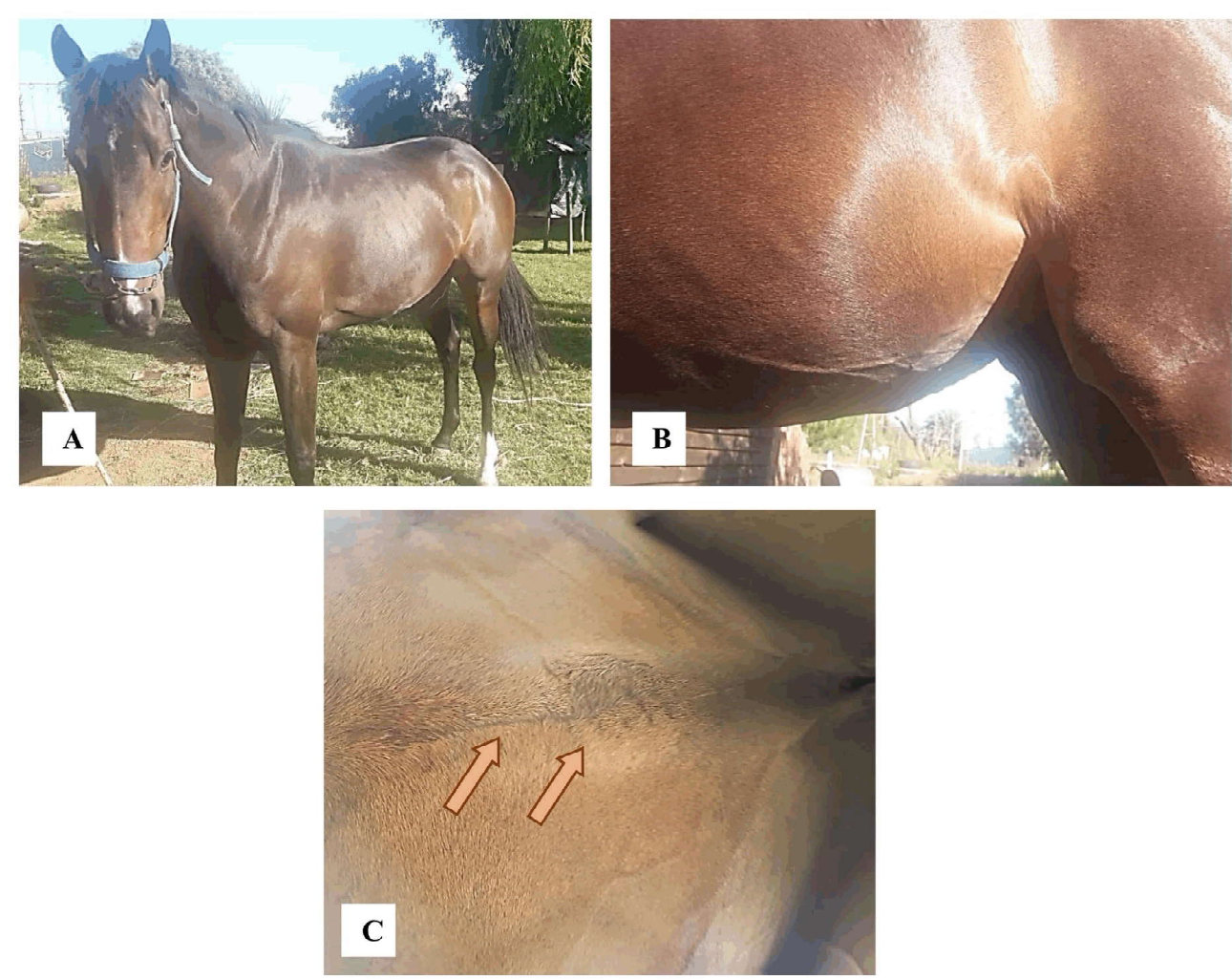

Figura 5. Potranca Pura Sangre de (arrera a los 60 días postoperatorio (A); vista lateral del abdomen (B); Zona de la intervención totalmente recuperada (C)

tida mediante cuerdas de la cabeza y cola. Se administró fenilbutazona (Butaflex, Richmond Veterinaria, Argentina) $2.2 \mathrm{mg} / \mathrm{kg}$ vía IV cada 12 horas por 3 días, penicilina procaínica (penicilina G sódica, Equi Systems, Argentina) IM a dosis de $20000 \mathrm{UI} / \mathrm{kg}$ por 5 días; gentamicina (Gentamic 10\%, Laboratorios Microsules, Uruguay) $6.6 \mathrm{mg} / \mathrm{kg} \mathrm{IM}$ por 3 días y flunixin meglumine (Fluxamine, Ripoll, Uruguay) a $0.25 \mathrm{mg} / \mathrm{kg}$ IV cada 8 horas por 3 días. En cuanto al manejo alimenticio, el ayuno sólido fue por $24 \mathrm{~h}$; luego, se iniciaron sesiones de verdeo de 5 min cada 4 $\mathrm{h}$; a las $48 \mathrm{~h}$ se incorporó heno de alfalfa mojado luego del verdeo. A partir de las $96 \mathrm{~h}$ se inició el suministro de pequeños volúmenes de avena en grano humedecido, aumentando el forraje y grano progresivamente. El pronóstico del caso fue reservado hasta confirmar el normal tránsito intestinal. No se detectaron complicaciones posoperatorias (Fifura 5). El paciente permaneció internado por siete días, regresando a su lugar de origen con reposo de cualquier actividad deportiva y espacio restringido por un lapso de 60 días. La yegua no ha presentó ningún episodio de dolor abdominal ni de línea media y ya ha participado en competencias de carreras.

\section{Discusión}

Al examen ultrasonográfico de este tipo de patologías se suele observar el asa visceral con ondas peristálticas dentro del saco herniario, así como estructuras o sustancias como exudado o líquido ascítico (Middlebrook y Eftekhari 1992). En este paciente, el grado de inflamación y la presencia de pus no permitió diferenciar correctamente tales estructuras, por lo que se optó realizar una exploración quirúrgica, basado en que el dolor se originaba en la evidente inflamación y posible necrosis de un segmento intestinal adherido 
(Marshall y Blikslager 2012), como sucede en estos casos (Steinke y Zellweger 2000).

El diagnóstico de la hernia de Richter se obtuvo en el intraquirúrgico al hallar que la zona antimesentérica de un segmento del íleo estaba adherido y estrangulado en el saco herniario (Steinke y Zellweger, 2000). Este tipo de hernia se caracteriza por una gangrena focal en la pared intestinal que desencadena signos de inflamación con sensibilidad o hinchazón de la zona herniaria (Middlebrook y Eftekhari, 1992), aunque inicialmente parece inocua porque no llega a causar obstrucción intestinal (Steinke y Zellweger, 2000).

En este caso, a través de la herida quirúrgica se pudo eliminar el contenido purulento del saco herniario según procedimiento estándar. Otro hallazgo quirúrgico fue la evidencia de depósitos de fibrina y adherencia de la zona antimesentérica debida a la inflamación (Marshall y Blikslager, 2012). Esto indica que se había ido desarrollando cierto nivel de lesión (Gorvy et al., 2008) que desencadenó la fisiopatología que conduce a la generación de adherencias (Alonso et al., 2014), probablemente por la persistencia de la hernia en el tiempo (Steinke y Zellweger, 2000). La presencia de la adherencia ileal sumado al proceso infeccioso debió desencadenar la inflamación, coagulación y reparación (Trigo y Valero, 2004), pero estos procesos no fueron eficaces dada la persistencia de la noxa.

La enterotomía se realizó con la finalidad de retirar la zona adherida y necrótica (Figura 3); sin embargo, la posibilidad de generar una estenosis quirúrgica de la luz ileal era inminente, a lo que se sumaba la presencia de edema en la pared intestinal que no permitiría ejercer la tensión con patrones de sutura invaginantes en dos capas (Freeman, 2012). Dado que no hay diferencias en las complicaciones posoperatorias y en la supervivencia al usar suturas en intestino delgado con un patrón de sutura de una sola capa o de doble capa (Close et al., 2014), se eligió realizar una sutura continua simple con un revestimiento de la línea de sutura transponiendo el ligamento ileocecal (Figura 4), para que funcione como lo harían las bioprótesis autólogas (Pérez et al., 2005) o heterólogas, que son usadas para evitar las fallas anastomóticas (Testini et al., 2014; Aguilar et al., 2018), junto a otras técnicas quirúrgicas resumidas por Hansen y Monnet (2013).

La finalidad del revestimiento es garantizar la remodelación y evitar las fallas anastomóticas, sirviendo como barrera de defensa (Testini et al., 2014; Aguilar et al., 2018a), además de disminuir la probabilidad de generar adherencias en la línea de sutura (Aguilar et al., 2018b). Este procedimiento no trajo complicaciones en el posoperatorio, sino por el contrario, reforzó la línea de sutura.

\section{Literatura Citada}

1. Aguilar GC, Delgado CA, Chavera CA, Cueva RM. 2018a. Evaluación anatómica e histopatológica de la anastomosis yeyunal equina revestida con xenoinjerto de pericardio bovino. Rev Inv Vet Perú 29: 1101-1113. doi: 10.15381/ rivep.v29.14.15306

2. Aguilar C, Delgado A, Chavera A, Cueva M. 2018b. Anastomosis yeyunal término-terminal con xenoinjerto de pericardio bovino en equinos. Rev Inv Vet Perú 29: 106-119. doi: 10.15381/ rivep.v29i1.14190

3. Alonso JM, Garcia AL, Watanabe MJ, Rodrigues CA, Hussni CA. 2014. Peritoneal response to abdominal surgery/ : the role of equine abdominal adhesions and current prophylactic strategies. Vet Med Int 2014: 279730. doi: 10.1155/2014/279730

4. Blikslager AT, Carolina N, White NA, Moore JN, Mair TS. 2017. The equine acute abdomen. $3^{\text {rd }}$ ed. USA: Wiley. $904 \mathrm{p}$. 
5. Bristol DG. 1994. Enterocutaneous fistulae in horses: 18 cases (1964 to 1992). Vet Surg 23: 167-171. doi: 10.1111/ j.1532-950x.1994.tb00465.x

6. Close K, Epstein KL, Sherlock CE. 2014. A retrospective study comparing the outcome of horses undergoing small intestinal resection and anastomosis with a single layer (lembert) or double layer (simple continuous and cushing) technique. Vet Surg 43: 471-478. doi: 10.1111/j.1532-950X.2014.12143.x

7. Freeman D. 2012. Small intestine. In: Equine surgery. $4^{\text {th }}$ ed. Elsevier. p 416-453.

8. Freeman DE. 2019. Jejunum and ileum. In: Auer JA, Stick JA, Kümmerle JM, Prange T (eds). Equine surgery. $5^{\text {th }}$ ed. USA: Elsevier. p 536-575.

9. Hansen LA, Monnet EL. 2013. Evaluation of serosal patch supplementation of surgical anastomosesin intestinal segments from canine cadavers. Am J Vet Res 74: 1138-1141. doi: 10.2460/ ajvr.74.8.1138

10. Kummer MR, Stick JA. 2012. Abdominal hernias. In: Auer JA, Stick JA(eds). Equine surgery. $4^{\text {th }}$ ed. St. Louis, USA: Elsevier. p 506-513.

11. Lavoie JP, Hinchcliff KW. 2008. Blackwell's five-minute veterinary consult: equine. $2^{\text {nd }} e d$. USA: John Wiley \& Sons. $912 \mathrm{p}$.

12. Marshall JF, Blikslager AT. 2012. Colic: diagnosis, surgical decision, and preoperative management. In: Equine surgery. $4^{\text {th }}$ ed. St Louis, USA: Elsevier. p 402-407.

13. Martis JJS, Rajeshwara KW, Shridhar MK, Janardhanan D, Sudarshan S. 2011. Strangulated richter's umbilical hernia - a case report. Indian J Surg 73: 455-457. doi: 10.1007/ s12262-011-0272-Z
14. Middlebrook MR, Eftekhari F. 1992. Sonographic findings in Richter's hernia. Gastrointest Radiol 17: 229-230.

15. Neil KM. 2011. Disorders of the umbilicus and urachus. In: McKinnon AO, Squires EL, Vaala WE, Varner DD (eds). Equine reproduction. $2^{\text {nd }}$ ed. USA: John Wiley \& Sons. p 623-631.

16. Pérez D, Sotres A, Olmos R, Villalba $J$. 2005. Uso del pericardio bovino tratado con glutaraldehído. Rev Inst Nac Enf Resp 18: 224-229.

17. Ramírez JL. 2013. Hernia de Richter. Rev Méd Cos Cen 70: 77-79

18. Smith M. 2006. Management of umbilical disorders in the foal. In Practice 28: 280287. doi:10.1136/inpract.-28.5.280

19. Smith B. 2015. Large animal internal medicine. $5^{\text {th }}$ ed. USA: Elsevier Mosby, $2024 \mathrm{p}$.

20. Steinke W, Zellweger R. 2000. Richter's hernia and Sir Frederick Treves: an original clinical experience, review, and historical overview. Ann Surg 232: 710-718. doi: 10.1097/ 00000658-200011000-00014

21. Testini M, Gurrado A, Portincasa P, Scacco S, Marzullo A, Piccinni G, Lissidini G, et al. 2014. Bovine pericardium patch wrapping intestinal anastomosis improves healing process and prevents leakage in a pig model. Plos One 9: e86627. doi: 10.1371/journal.pone. 0086627

22. Tóth F, Schumacher J. 2019. Abdominal hernias. In: Auer JA, Stick JA, Kümmerle JM, Prange T (eds). Equine surgery. $5^{\text {th }}$ ed. USA: Elsevier. p 645-659.

23. Trigo FJ, Valero G 2004. Patologia general veterinaria. $4^{\circ}$ ed. México: Univ. Nacional Autónoma de México. 439 p.

24. Wilson DA. 2012. Clinical veterinary advisor: the horse. USA: Elsevier. $1104 \mathrm{p}$. 